\title{
Analysis and Simulation of Elastomeric Strut Mount and its applications for Failure Investigation
}

\author{
Nikita Hardikar*, Aadish Kulkarni, Adwait Telang and Vilas S.Kanthale \\ Mechanical Department, MIT College of Engineering, Savitribai Phule Pune University, Pune, India
}

Accepted 02 March 2016, Available online 15 March 2016, Special Issue-4 (March 2016)

\begin{abstract}
This paper gives an insight of elastomeric strut mount and the complexities experienced by the rubber material. Running vehicles are exposed to almost constant vibration excitation; shock absorbers are constantly required for reasons of driving safety and riding comfort. Strut is basically a shock absorber mounted inside a coil spring. Macpherson strut suspension consists of top rubber mount which helps in isolation of road vibrations and noise from entering passenger compartment. Poor design can cause excessive vibration and noise problem. Stress analysis of rubber components is often demanding on account of difficulties posed by complex behavior of rubber. When it comes to fatigue predictions of rubber components, this complexity increases multifold. Further, in time bound development projects, it becomes very difficult to dedicate sufficient time to tests and gather data required to characterize rubber accurately. In such cases, more practical approach is to make design improvements through simulations based on approximate rubber models followed by validation through physical tests.
\end{abstract}

Keywords: Strut mount, rubber, finite element analysis, simulation

\section{Introduction}

Damping is the process of absorbing energy of impacts transmitted through the forks or rear shock on the compression and rebound stroke. A set of forks, shocks or struts are considered dampers. They slow the rate at which the suspension absorbs or releases energy. Damping is necessary to prevent uncontrolled spring oscillations in the suspension. The introduction of damping will absorb some or all of the energy imparted to the suspension by a bump. The oscillation will be reduced or eliminated, depending on the degree of damping. The up and down (kinetic) energy absorbed by the spring is restricted as oil flows through a valve in the damper body, resulting in hydraulic friction. This hydraulic friction creates heat, (thermal energy). This is why hard worked suspensions, such as in motocross, sometimes overheat.

Struts have a very important secondary function. They are an integral part of the suspension. While a shock can be removed with only minor consequences, when a strut is removed the suspension will collapse, unable to hold the car off the ground once the important link provided by the strut is removed.

MacPherson strut suspension systems typically utilize either a steering knuckle or a hub carrier that has two mounting points that attach it to the body of the vehicle. The lower mounting point attaches to a track control arm or lower control arm, and it is this connection that dictates both the longitudinal and lateral orientation of the wheel assembly. In turn, the upper mounting point of the knuckle or hub is attached in some way to an assembly that contains a coil spring and a shock absorber. It is this combination of housing, spring, and dampener that is referred to as a strut or, more properly, as a MacPherson Strut.

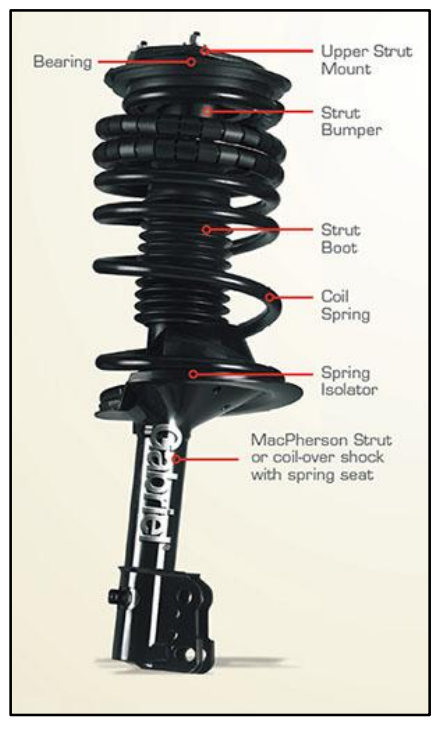

Figure 1 MacPherson Strut 


\subsection{Problem statement}

During design phase it is important to predict failure of rubber components and to propose design improvements in-line with other metal components. Poor design can cause excessive vibration and noise problem. Therefore it is important to validate strut mount design using finite element analysis, before proto testing.

Finite element analysis of Strut mount is challenging since rubber exhibits highly non linear behaviour. Simulation of rubber requires proper material modelling and simulation tools which are entirely different from those traditionally used for metals. This work proposes appropriate material model and simulation tool for FE analysis of strut mount.

\subsection{Objective}

Stress analysis of rubber components is often demanding on account of difficulties posed by complex behavior of rubber. When it comes to fatigue predictions of rubber components, this complexity increases multifold. Further, in time bound development projects, it becomes very difficult to dedicate sufficient time to tests and gather data required to characterize rubber accurately. In such cases, more practical approach is to make design improvements through simulations based on approximate rubber models followed by validation through physical tests. Correct choice of hyper-elastic material model that is to be derived from limited test data is a key to success of this approach. It is seen that incorrect choice of material model may lead to erroneous results and misguide design changes.

\subsection{Rubber models}

Rubber material usually has long chain molecules recognized as polymers. The term elastomer is the combination of elastic and polymer and is often used interchangeably with the rubber. In recent years, rubber component as an engineering material has been used in many industries such as automotive and in a wide range of applications consist of engine mountings, tires, vibration isolators, medical devices and structural bearings. Rubber is an ideal material for many applications because it can withstand very large strains over $500 \%$ with no permanent deformation or fracture. Besides elastic recovery, elastomers have special physical properties (flexibility, extensibility, resiliency and durability), which are unmatched by other types of materials; however it still presents behavior in common with other material.

\subsection{Mullins effect}

Stress softening is known as the Mullins effect and happens during the initial loading cycles. It is closely related to the fatigue of rubber components used in engineering applications.
Softening occurs if the material is cyclically deformed, that is, a lower force is needed during the second loading cycle to the maximum amplitude $\gamma 0$ in comparison to the first cycle (path 3 versus path 1 ). If the amplitude is increased beyond $\gamma 0$, the loading curve (path 4) follows the path of a single loading experiment (black dashed line). If the material is cyclically loaded to a new maximal amplitude $(\gamma 1$ or $\gamma 2$ ), again softening is observed (path 6 versus path 4 , or path 9 versus path 7 ).

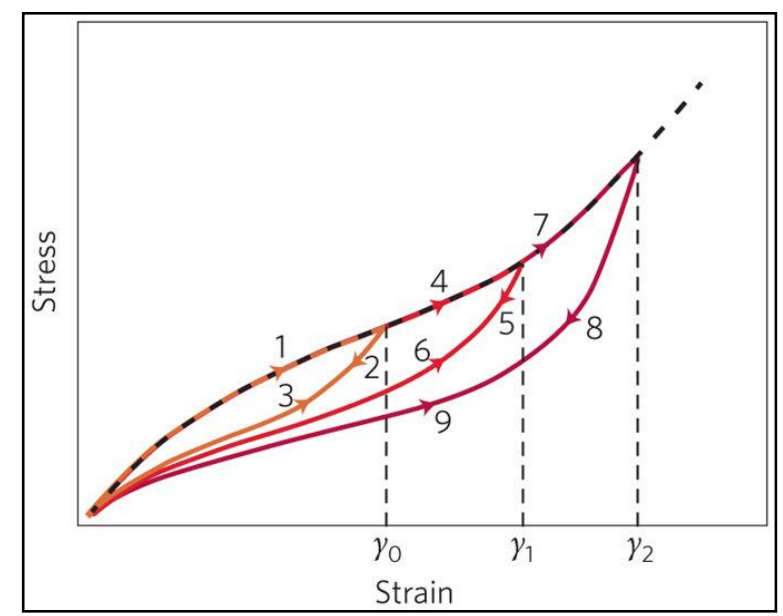

Figure 1.4Mullins Effect

\subsection{Hyperelastic models}

The typical elastomer has a non linear stress strain curve. A hyperelastic material definition requires a function, in particular a strain energy dendsity function. A considerable amount of literature has been published on modeling of rubber material. The choice of the model depends to its application, corresponding variables and its available data to determine material parameters. The validity of possible models should be studied and the simplest is selected with high accuracy and low materials parameters. The modeling and design of hyperelastic materials is the selection of a proper strain energy function $\mathrm{W}$, and accurate determination of material constants for function. There are various forms of strain energy potentials for modeling of incompressible and isotropic elastomer.

An efficient hyperelastic model can be explained by four main qualities:

- It should have the ability to exactly reproduce the whole 'S' shaped response of rubbers

- The change of deformation modes must not be problematic, i.e., if the model operates sufficiently in uniaxial tension, it must also be exact in simple shear or in equibiaxial tension

- The number of fitting material parameters should be small, in order to decrease the number of experimental tests for their determination

- The mathematical formulation should be simple display practicable for the numerical performance of the model. 


\subsubsection{Mooney Rivlin}

In continuum mechanics, a Mooney-Rivlin solid is a hyperelastic material model where the strain energy density function $\mathrm{W}$ is a linear combination of two invariants of the left Cauchy-Green deformation tensor. The model was proposed by Melvin Mooney in 1940 and expressed in terms of invariants by Ronald Rivlin in 1948. The strain energy density function for an incompressible Mooney-Rivlin material is

$$
W=C_{1}\left(\bar{I}_{1}-3\right)+C_{2}\left(\bar{I}_{2}-3\right),
$$

where $C_{1 \text { and }} C_{2}$ are empirically determined material constants, and $I_{1}$ and $I_{2}$ are the first and the second invariant of the unimodular component of the left Cauchy-Green deformation tensor

Strain energy potential is proposed:

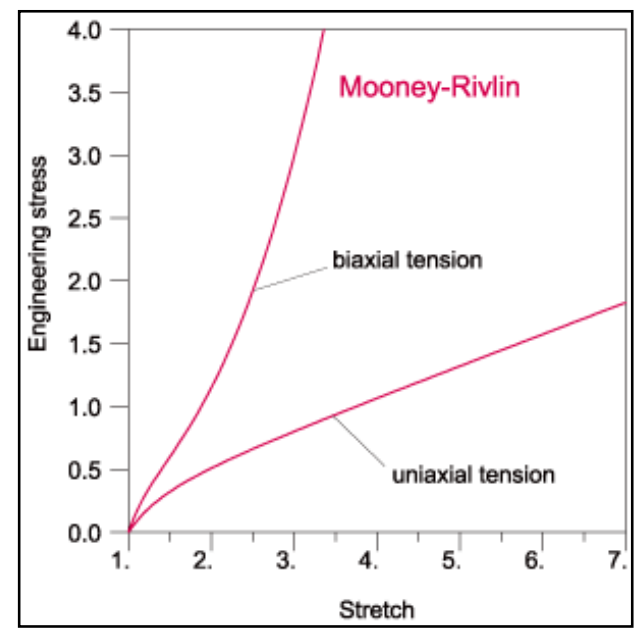

Figure 1.5.1 Mooney Rivlin curve for uniaxial and biaxial tension

The most favorite ones of constitutive models are Mooney-Rivlin and Ogden models. Their disadvantage is that the material parameters must be obtained by experiments and they are not physically-based parameters. The fitting method can be complicated if the number of parameters is large.

\subsubsection{Yeoh Model}

[The Yeoh strain energy function is presented as following when $\mathrm{N}=3$ in the Reduced Polynomial model

$$
\mathrm{U}=\sum_{\mathrm{i}=1}^{3} \mathrm{C}_{\mathrm{i} 0}\left(\overline{\mathrm{I}}_{1}-3\right)^{\mathrm{i}}+\sum_{\mathrm{i}=1}^{3} \frac{1}{\mathrm{D}_{\mathrm{i}}}\left(\mathrm{J}_{\mathrm{el}}-1\right)^{2 \mathrm{i}}
$$

The initial shear modulus and bulk modulus are given by:

$$
\mu_{0}=2 C_{10}, K_{0}=\frac{2}{D_{1}}
$$

For following reasons this model has been chosen to describe the hyperelastic properties of rubber compounds

- Yeoh model is applicable for a much wider range of deformation

- This model is able to predict the stress-strain behavior in different deformation modes from data gained in one simple deformation mode like uniaxial extension.

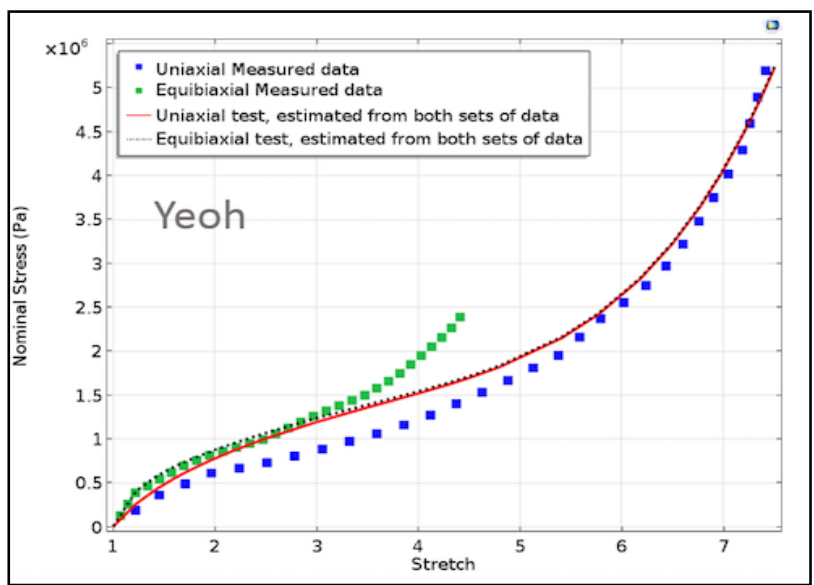

Figure 1.5.2 Nominal stress v/s stretches for Yeoh model

\section{Literature Review}

Prashant Chavan, Amol Apte, TATA motors Itd. Simulia (2011)[6] have carried out failure analysis and simulation using CAE of strut top bush by considering axi-symmetrical meshing.

CAE and test results are discussed in detail. While working with limited test data, it has been decided to work with multiple material models to assess design improvements with minimum risks. This hybrid testCAE approach was proved to be successful in making design improvements in very short period of time with limited test support.

\section{Experimentation}

For the purpose of meshing and simulation, we first prepared a CATIA model of the strut top mount pertaining to the existing design. This strut top mount is used in the standard MacPherson strut. The CATIA model is based on the dimensions and tolerances considering the normally used natural rubber.

The top mount consists of upper bracket, insulator plate, rubber component, lower bracket and bearing cup.

The deformation of rubber component is kept in check by the insulator plate and this whole assembly experiences different loading conditions. The existing design uses natural rubber which is leading to improper functioning of the strut mount as a result of insufficient space for the rubber to obey its elastic nature and heat is dissipated on a large scale. 


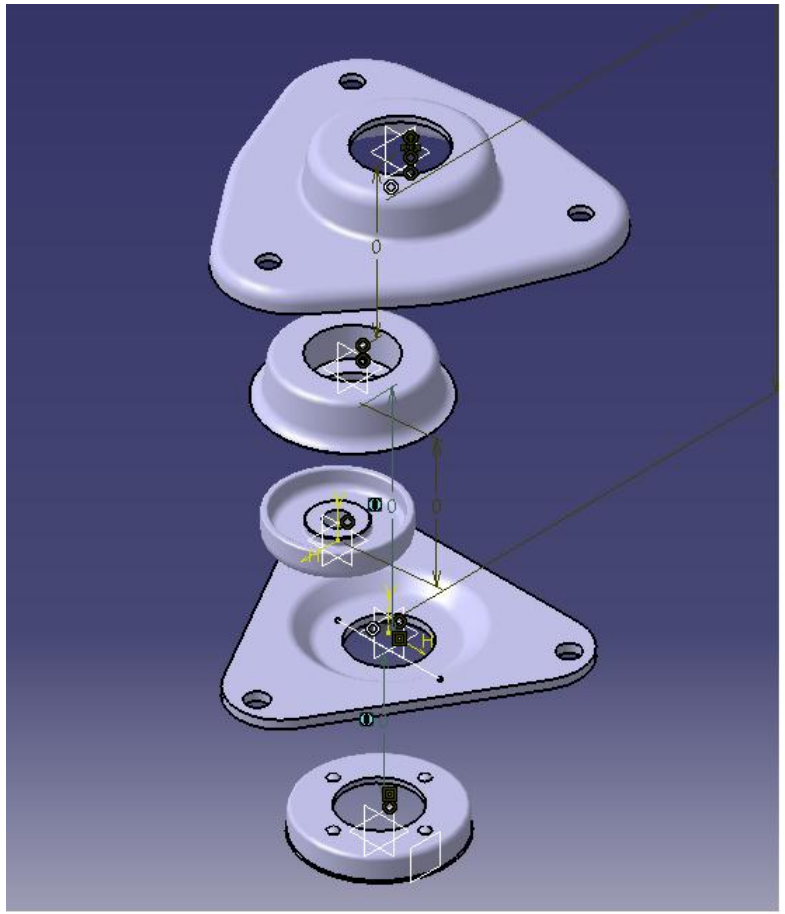

Figure 3.1 Exploded view of strut top mount

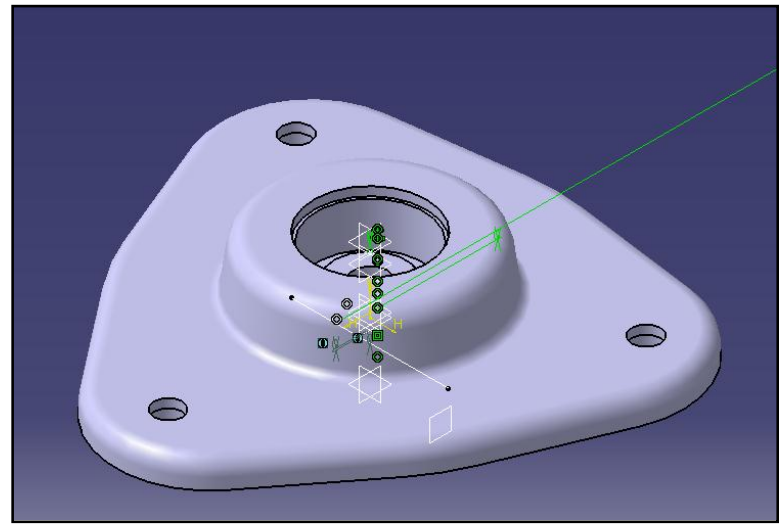

Figure 3.2 Assembly of strut top mount

\section{Meshing}

The CATIA model of strut top mount had to be meshed uniformly for further simulation regarding stress concentration using ANSYS. We used the HYPERWORKS software for meshing the component. This meshed component imported in ANSYS, along with the material model constants and specific load application will give us the stress concentration on the rubber component.

The model of strut top mount was imported in the

HYPERWORKS software and meshed using Hypermesh. For ease of meshing, we took a sectional view of the top mount and separately meshed each part with respect to other. Uniform meshing was achieved by adding more number of nodes at curved parts.
The feature of revolving the mesh on one plane of the component is available in Hypermesh which proved to be advantageous in achieving uniform meshing for the entire component.

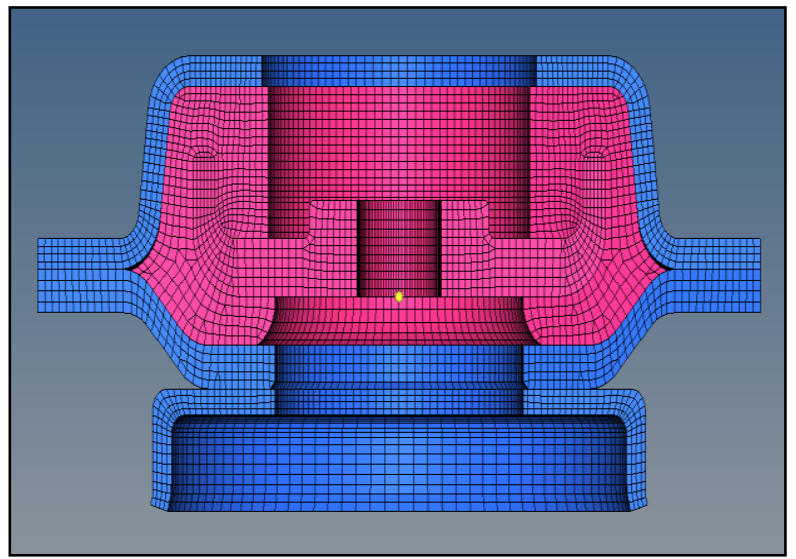

Figure 4 Sectional meshed view of the top mount

\section{Selection of hyper elastic rubber model}

In order to perform simulation in ANSYS for determining the stress concentration on rubber, a particular rubber model needs to be selected. For this purpose, data obtained from uni-axial testing of natural rubber was used for curve fitting using ANSYS.

The following table consists of stress strain values obtained from AXEL products uniaxial testing of natural rubber.

Table 5 Uniaxial test data for natural rubber

\begin{tabular}{|c|c|}
\hline Strain & Stress \\
\hline 0.98102 & 2.8104 \\
\hline 0.98328 & 2.81879 \\
\hline 0.98567 & 2.8285 \\
\hline 0.98818 & 2.83667 \\
\hline 0.99018 & 2.84461 \\
\hline 0.99256 & 2.853 \\
\hline 0.99495 & 2.86227 \\
\hline 0.99707 & 2.87132 \\
\hline 0.99972 & 2.87948 \\
\hline
\end{tabular}

We used ANSYS for the purpose of curve fitting. In ANSYS, under preprocessor and material models, we selected nonlinear hypermesh and curve fitting. After importing the values pertaining to stress strain, we obtained the values of constants and respective plots.

Following are the results obtained by using Yeoh model and Mooney-Rivlin model

YEOH (5 parameters) 


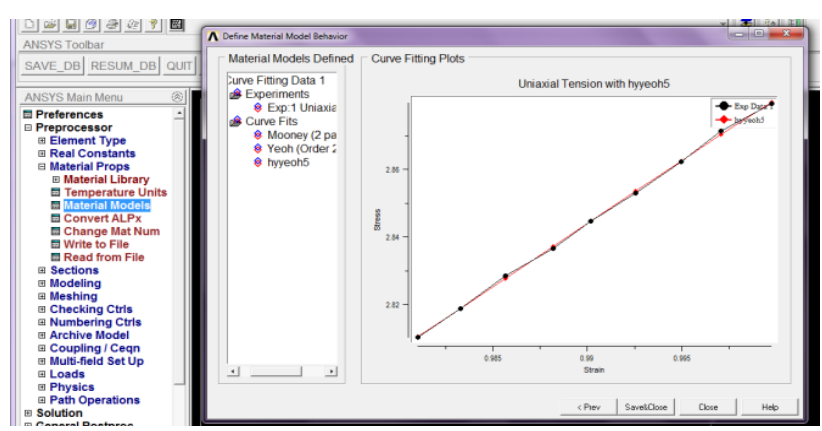

Figure 5.1 Curve fitting plot of Yeoh with 5 parameters

Values of constants

C_10 $=372.363949817$

C_20 $=-371.602718555$

C_30 $=185.677424388$

C_40 = -46.3575911353

C_50 = 4.62729843285

Mooney-Rivlin (2 Parameters)

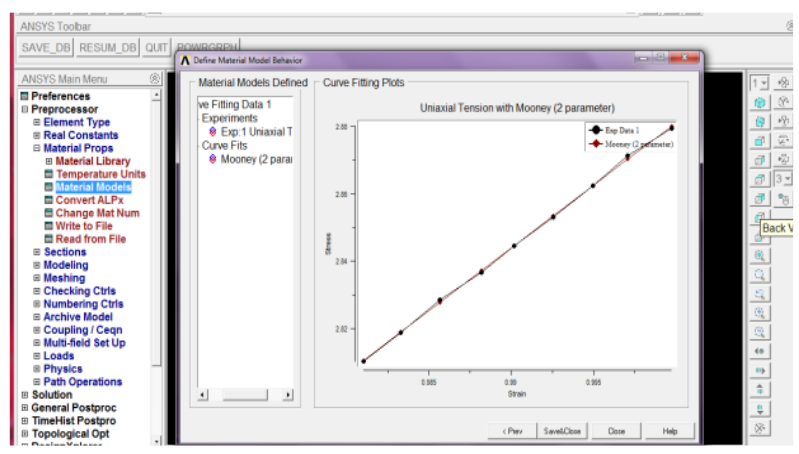

Figure 5.2- Curve fitting plot of Mooney Rivlin

Values of constants

$\mathrm{C} 10=1.77408699001$

$\mathrm{C} 01=-1.90181373363$

\section{Results and Discussion}

Overall study of strut mount and its practical applications has been done. The existing design has been analyzed and only simulation of the rubber is yet to be done.
By acquiring the stress concentration on the existing piece of natural rubber and the top mount, further changes in design need to be done. It is yet to be seen how rubber behaves after a force acts on it. By correlation and analysis the design maybe altered for better results and fatigue investigation on the component.

\section{Acknowledgements}

We would take this opportunity to extend our heartfelt gratitude towards Gabriel India Ltd, for their overall sponsorship for our research work.

\section{References}

Scott Allen Noll, Benjamin Joodi, Jason Dreyer, and Rajendra Singh, (2015), Ohio State University, Volumetric and Dynamic Performance Considerations of Elastomeric Components, SAE international

Mary. C. Boyce, Ellen Arruda, (2000), Constitutive Models of Rubber Elasticity

Hutton DV, (2004) ,Fundamentals of Finite Element Analysis - McGraw-Hill

Norton M.P., Karczub D.G.,( 2003), Fundamentals of Noise and Vibration Analysis for Engineers, Cambridge university press.

Kurt Miller, (2001), Using slow cyclic loadings to create stress strain curves for input into hyper elastic curve fitting routines, Cyclic loading revC

Prashant Chavan, Amol Apte, (2011), Failure analysis of strut top rubber bush \& sensitivity study using multiple material models, Tata Motors, SIMULIA India Regional General Meeting

Jörnsen Reimpell, Helmut Stoll and Jürgen W. Betzler, The Automotive Chassis (Second Edition) http://www.axelproducts.com http://www.polymerFEM.com http://www.gabrielindia.com ABAQUS 6.6 documentation: Mullins Effect in Elastomers Ayman A. Aly, January (2012), Car Suspension Control Systems: Basic Principles, International Journal of Control, Automation and Systems Vol.1, No.1

G. Thomas Mase, Continuum Mechanics, CRC Press. 